\title{
Fabrication and Testing of Miniature Automatic Photophoretic Trapping Rigs
}

\author{
Riley Kuttler ${ }^{1}$, Dylan Barton ${ }^{1}$, Brenden Weaver ${ }^{1}$, Alexander Steffan ${ }^{1}$, Braden Huffman ${ }^{1}$, Steven Griffith $^{1}$, Daniel Smalley ${ }^{1}$ \\ ${ }^{1}$ Electrical Engineering, Brigham Young University
}

\section{Corresponding Author}

Riley Kuttler

rileykkuttler@byu.edu

\section{Citation}

Kuttler, R., Barton, D., Weaver, B., Steffan, A., Huffman, B., Griffith, S., Smalley, D. Fabrication and Testing of Miniature Automatic Photophoretic Trapping Rigs. J. Vis. Exp. (177), e63113, doi:10.3791/63113 (2021).

\section{Date Published}

November 23, 2021

DOI

$10.3791 / 63113$

URL

jove.com/video/63113

\section{Abstract}

This paper presents an automated, rapid-fab-compatible, photophoretic trap test rig to enable the democratization and crowdsourcing of volumetric display research. The rig can be constructed within $2 \mathrm{~h}$ using a laser cutter, 3-dimensional (3D) printer, and common hand tools. In its current form, the rig can be used to test the following critical parameters: particle type, trap type, numerical aperture, and airflow at a rate of approximately 250 samples per hour. With minor modification, the rig can be made to test an even larger set of parameters, such as laser power and laser wavelength, depending on the user's needs. The rig can use machine vision for automated data capture and analysis. The operation and construction of the test rig are described with concise, easy-to-follow steps. Results from a four-unit test rig 'farm' covering the power and particle type parameters are reported. This platform will broaden the scope and composition of optical trap display parameters and researchers through accessibility and democratization.

\section{Introduction}

The optical trap display (OTD) makes possible the display geometries seen in science fiction. It operates by trapping a particle through photopheresis and illuminating the particle ${ }^{1,2,3,4}$. Then, dragging that particle through space forms an image in the air that the viewer perceives as continuous per the persistence of vision ${ }^{5}$. This screenless 3D technology allows it to display geometries such as long-throw projections, tall sand tables, and wrap-around displays ${ }^{1}$. These geometries are uniquely compelling because they require no screen and create content that can be seen from virtually every angle.

Researchers at Brigham Young University found initial success in their first-generation photophoretic trapping system by using a beam expander and galvanometer scanners, along with several mirrors and one or more spherical lenses to create a photophoretic trap through spherical aberration ${ }^{1,4}$. This first-generation trapping rig also contained RGB (red-green-blue) lasers to allow for precise colored display illumination. Using this trapping system, OTDs 
are created by moving a single particle through a convoluted path. This approach limits the size of images to under a cubic centimeter and limits the complexity of real-time images to wireframes and other sparse content ${ }^{6,7}$. Furthermore, the scaling of this technology is limited by the inconsistency of photophoretic trapping ${ }^{8}$. If a single trap/particle system can be optimized, scaling the display could be achieved by replicating an optimized trap and synchronously trapping and scanning multiple particles ${ }^{9}$. Any problems with a single trap will be compounded in a multi-trap system, so careful optimization of trap and particle parameters is critical.

The optimization of an individual trap/trapping system requires extensive testing to be done for every parameter of the photophoretic trapping system ${ }^{7}$. Such parameters include particle type (substance, shape, size), laser power, laser wavelength, and numerical aperture (focal length, diameter, tilt). Testing and experimentation through trial and error for each parameter will optimize individual traps and multiple synchronous traps. Still, they will require large amounts of data to be collected.

In the past, the research and testing process for optimizing photophoretic trapping through spherical aberration has only been done by a handful of researchers across the world $^{1,2,3,4,5,6,7,8,9,10}$. Until recently, researchers at Brigham Young University have relied on a single, large, expensive trapping system to collect the data needed, which caused the process of testing and gathering data to be slow $^{1,7}$. However, since introducing optical trap displays as a solution for $3 \mathrm{D}$ visualization in $2018^{1}$, individuals of all age groups and from several continents have expressed a desire to participate in the research. Because of the generated interest in OTDs, researchers have wanted to find a way to allow all interested parties to participate in the research process. Previous generations of photophoretic trapping rigs, which contained beam splitters and galvanometers, were too expensive and time-consuming to mass-produce and crowdsource $^{1,6}$, so a different solution was needed.

A new miniature photophoretic trapping rig has been developed, which allows all interested parties to participate in research and quickly test and gather data for all significant parameters mentioned above. They can be fabricated rapidly by anyone who has access to a 3D printer and laser cutter. This design attempts to minimize cost and complexity, mitigate risk, and maximize automation, interconnectivity, and flexibility (Figure 1). The new rig employs the most straightforward optical setup for photophoretic trapping possible: a single laser and lens ${ }^{10}$. The small rigs are simple to use once set up and can test at a rate of approximately 250 attempts per hour.

The data collected from these rigs from the tests of future citizen scientists and researchers will significantly help develop photophoretic trapping in its use for 3D visualization by allowing for the optimization of trapping parameters and individual traps.

\section{Protocol}

\section{3D printing and laser cutting of the required materials}

1. 3D print the materials listed below

1. Print the lens holder using a filament FDM (Fused Deposition Modeling) 3D printer (see Table of Materials) as per the information provided in Supplementary File 1. This lens holder is for a 30 $\mathrm{mm}$ (diameter) lens (Figure 2). 
NOTE: The file can be easily customized to fit other lenses.

2. Next, print out the cantilever platform and the holder (Figure 2) (Supplementary File 2 and Supplementary File 3).

NOTE: This can take $\sim 2-14 \mathrm{~h}$, depending on the detail of the prints. The detail of the prints should not affect the trapping rate if enough of the trapping substance has been placed (step 5.3.4).

2. Using a 3D laser cutter (see Table of Materials), cut out the rig pieces (Figure 3) (Supplementary File 4-5).

NOTE: This cut can be done on any material of quarterinch thickness, but wood is the recommended material.

Supplementary File 4 contains the required rig pieces that fit on a $12 \times 12$ in piece of wood. Supplementary File 5 includes an optional light shield/blocker.

3. Using a glass cutter (see Table of Materials), cut a standard test tube $(-2.5 \mathrm{~cm}$ in diameter) roughly in half so that the half with two open ends is $\sim 6.5 \mathrm{~cm}$ long.

\section{Assembling the wooden rigs}

NOTE: While assembling the wooden rig, instructions in steps 2.1 -2.5 may say "slide," but pieces may require more force to be appropriately positioned and built.

1. Place the base piece down with the $Y$ emblem facing up (Figure 1A).

2. Hold the two long side pieces on either side of the base while the first laser holder is slid in to place on one end and the first test tube holder on the other side (Figure $1 \mathrm{~B}, \mathrm{C})$.

3. Off to the side, slide both electromagnet holders onto the camera holder. Ensure that the magnet holders are separated by $\sim 1 \mathrm{~cm}$ on each side (Figure 1D).
1. Slip the magnet holders and the camera holder as a unit next to the first test tube holder, spaced. Hence, $1 \mathrm{~cm}$ between the test tube holder and the first electromagnet holder (the edge of the camera holder past the electromagnet holder can serve as a reference for $1 \mathrm{~cm}$ ) (Figure 1E).

NOTE: Both the camera holder and light shield are not required for the primary use of the miniature trapping system, but both are recommended for the uniformity of the rig setup.

4. Next, place the second test tube holder after both electromagnet holders so that there is $\sim 1 \mathrm{~cm}$ space between the second test tube holder and the second electromagnet holder.

NOTE: In total, between the two test tube holders, there is $\sim 4 \mathrm{~cm}$ of space (the camera mount has a width of 4 $\mathrm{cm}$, for a positioning reference) (Figure 1F).

1. If using the optional light shield/blocker (Supplementary File 5), slide the light shield onto the opposite end of the test tube holders and electromagnet holders from the camera holder; this will help center and align the test tube and electromagnet holders.

5. Slide the second laser holder into place; no exact distance is required. The recommended distance is 3-4 $\mathrm{cm}$, but this may need to vary depending on the length of the laser.

6. If desired, an optical rail (see Table of Materials) can be slid under all the holders to align other elements of the trapping system. This will be particularly useful for aligning the lens with the laser and test tube (Figure 1G).

7. Place the electromagnet (see Table of Materials) into the electromagnet holders (Figure 1H). 


\section{Connection of the circuit with the specified microcontroller board}

1. Plug in a computer monitor, keyboard, and mouse to the microcontroller board (see Table of Materials). Startup the microcontroller board and ensure that the operating system is functioning. No changes in the original microcontroller configurations are required, although both VNC (virtual network connection) and SSH (secure shell) can be selected if desired. This would allow remote access to the microcontroller.

2. Build the electromagnet controlling circuit using a voltage regulator (see Table of Materials), a breadboard, and a few wires (Figure 4A).

NOTE: All pin numbers for the microcontroller board are the GPIO (general purpose input and output) pins.

1. Place the voltage regulator in the breadboard so that each pin is in a different row to allow for proper usage.

2. Wire the input pin of the voltage regulator to one of the $5 \mathrm{~V}$ power pins on the microcontroller board.

3. Wire the adjust pin of the voltage regulator to GPIO 23 on the microcontroller board.

4. Connect the input wire of the electromagnet to the output pin of the voltage regulator. Then connect the output wire of the electromagnet to a ground pin on the microcontroller; this is best achieved when using another row in the breadboard to connect the two using an additional wire.

\section{Uploading the code for operating the system}

NOTE: For uploading the code, either step 4.1 or step 4.2 needs to be followed. Step 4.1 provides instructions for the simple version of the code that does not use a camera. Step 4.2 provides instructions for the version that uses a camera.

1. Perform the steps in alignment with the instructions provided in Supplementary File 6.

1. Open the terminal and navigate to the location to store the needed files. Create a new directory on the microcontroller board by typing the terminal command 'mkdir' followed by the desired directory name. This directory will be used to store the files for running the trap rig.

2. Insert Supplementary File $\mathbf{6}$ into the new directory. See the readme section at the beginning section of the file for details. After changing the test number to the desired amount, the program is ready to run. NOTE: This file contains one needed variable called num_tries which controls how many tests to do in one run. This file always includes a few pauses, which can be shortened for quicker tests.

2. Run and view SQLite on the microcontroller board following the steps below. This requires Supplementary File 7-11 and a camera and the relevant expertise.

1. Install the needed database libraries onto the microcontroller board by typing in the terminal 'Sudo apt-get install SQLite browser' and 'Sudo apt-get install sqlite3'. This will allow the microcontroller board to automatically store all data from the tests using Supplementary File 9.

2. Save Supplementary File 11, the camera script as main.py on the camera. This can be done through a file explorer or the integrated development environment (IDE) developed for the camera (see Table of Materials). 
NOTE: IDE is recommended because it allows users to see the camera's output, which helps when ensuring that the camera is focusing correctly.

3. Connect the camera to the microcontroller board. Use 4 pins, including the ground pin from the camera. Connect the ground pin must to the ground of the microcontroller board. The following pins should match as mentioned below:

1. Connect Camera pin 8 to GPIO 19: This pin sends the results of each trap back to the pi.

2. Connect Camera pin 9 to GPIO 17: This pin gives the camera permission to start searching.

3. Connect Camera pin 7 to GPIO 5: This is the camera status pin.

4. Create a directory to store all the files. After creating this directory, save the files as provided in Supplementary File 7-10 in the directory; rename them readme.txt, main.py, electromagnet.py, and test_insert.py, respectively.

5. Read through Supplementary File 7 (ReadMe.txt). NOTE: The readme file gives a good explanation of what each file does and the changes that might be needed on each file, such as the directory path of the database.

6. Open the Database viewer installed in step 4.2.1. Click the new database button and save the database in the same directory as the other files. The new database must match the name of the database file found in test_insert.py.

7. Inside the database, create a table inside the new database to save the data. The database has 5 fields, parameter_type, trapped, testname, testnum, and rigID.

NOTE: The table sections must be precise as specified or more changes will need to be made in Main.py and test_insert.py.

\section{Test Preparations}

1. Prepare the lens by placing the lens inside the lens holder. Ensure that the lens stays inside the holder during testing. Some hot glue might be needed here.

NOTE: The rig requires using a spherical bi-convex to ensure the proper formation of trapping regions.

2. After preparing the lens (step 5.1), place the lens holder on the optical rail and the laser (see Table of Materials) in the laser holder.

NOTE: The suggested laser in the list of materials requires no calibration before use. Safety glasses must be worn anytime the laser is in use.

1. Using the lens and the laser or other light source, find the focal point of the laser and slide the lens holder along the optical rail until the focal point is centered over the electromagnet.

NOTE: This step is critical for trapping; if the focal point is not centered over the electromagnet, the cantilever platform will not raise particles into the focal point.

2. Mark this point with a pencil on the wooden base for future reference.

NOTE: Each lens already comes with a focal length measurement, but these measurements are not always correct.

3. Prepare the trapping cite

1. Ensure that the laser is properly turned off again. 
2. Using a hot glue gun, glue a small button magnet (see Table of materials) of the same polarity as the electromagnet onto the flat surface of the platform so that the electromagnet will repel the platform.

NOTE: The polarity of the magnets must be matched appropriately so that the platform will be repelled by the electromagnet, pushing the particles into the beam of the laser so that trapping can occur.

3. Take the 3D-printed, cantilever-like platform and coat the platform in black aluminum foil, which protects the platform from melting.

NOTE: Regular foil can be used, but it causes too much glare for the camera system to be used. Try using the black foil tape (see Table of materials), which works fine with the camera (Figure 5A). Foil is recommended because it can be easily replaced for testing other substances, but similar products can be used if desired.

4. After covering the platform with the aluminum foil, place the selected particle type chosen by the user for testing on the slanted side of the platform (see Table of Materials for particle type options or see Figure 6A).

5. Gently insert the cantilever arms into the circular holder so that the magnet side is facing out. Then gently insert the test tube into the same holder. If this has been done correctly, the magnet will almost be touching the glass (Figure 5B).

6. Place the test tube onto the test tube holders so that the platform is centered over the electromagnet. If the magnet has been appropriately attached to the cantilever platform, the cantilever should appear to be in an upward position repelled by the electromagnet.

4. Place the camera in the camera holder to capture any traps that occur above/around the platform. Then doublecheck all other positionings (Figure 1I-J).

\section{Beginning the test}

NOTE: For testing, either step 6.1 or step 6.2 needs to be followed.

1. If using instructions from step 4.1, press start in the file or start the file normally from the terminal.

2. If using instructions from step 4.2, start this test from the terminal with the parameters as mentioned below.

1. Using terminal commands, run the file system once inside the proper directory with the command "python3 main.py test_num parameter_type exact_parameter". The file main.py is provided in Supplementary File 8.

2. Replace the test_num with the number of tests desired. Replace the Parameter_type by the type of parameter the test is focused on.

NOTE: For example, if tests were being done to determine what powered laser is best, Parameter_type would be replaced by laser_power, and the exact_parameter would be replaced by the optical output power of the current laser.

\section{Representative Results}

The principal result achieved by following the above protocol is creating a miniature photophoretic trapping rig, which can quickly test various parameters. So far, these rigs have been used to test two critical parameters, laser power, and particle type. The parallel running of multiple miniature rigs has 
allowed researchers to collect data with much larger sample sizes in a much quicker period of time.

The first test, conducted while developing the above protocol, was a laser power test. A single miniature rig was used during this test, without the camera detection system, as it had not yet been developed; instead, step 4.1 from the protocol was used. This required a researcher to be present for the data collection of all trap detections. The goal of this test was to determine the ideal laser power output for trapping to occur. By placing an optical attenuator (variable neutral density filter) between the laser and lens on the rig, the optical power of the laser was varried. Figure 7 shows the results of this experiment. A high optical power output corresponded with a higher rate of trapping. The laser at full power had the highest recorded trapping rate for this test. However, this test was limited to one laser with a maximum optical power of $\sim 120$ $\mathrm{mW}$.

The second test was to determine which material or substance would have the highest rate of trapping. This test was conducted using a singular miniature test rig without the camera detection system. Ten different particles were tested with a sample size of 100 attempts for each particle (all the substances tested can be found in the Table of Materials with their descriptions). The sample size was limited to 100 because of the need to have a researcher watch each attempt for data collection. All the necessary data was collected in two working days. Figure 6A shows the results of the particle type test. Of the 10 materials/particles types tested, it was found that diamond nanoparticles (55-75\%) and printer toner were the two best particle types with rates of $14 \%$ and $10 \%$, respectively (Table 1).

After the first two tests, researchers felt limited to a singular rig that required active watching during testing; this led to step 4.2 outlined in the protocol. This option includes a camera detection system, which enables users to run several miniature test rigs at a time and does not require a user present for testing.

For testing this new camera system, a modified re-test of the particle type test was conducted. Only a few different particle types were selected from the 10 used initially to be re-tested for this new particle type test. The selected particles underwent a new round of testing. Using a test rig "farm" of four miniature test rigs, each of the selected particles had a total test sample size of 4,000 attempts. Once again, all necessary data was collected in two full workdays (Table 2). The primary purpose of this particle type re-test was to test the new camera system. This test allowed for comparing results from the initial particle type test, with a researcher reporting the traps, to the results from the camera detection system. The test results were slightly different than the original tests but still comparable (Figure 6B). The best particle type from the initial test, diamond nanoparticles $55-75 \%$, was still the best in the re-test but did have a slightly lower trapping rate than before. The difference in results is most likely due to a bigger sample size and an imperfect camera detection system. Although the results for this particle test were slightly different than expected, when testing other parameters where the material stays constant, such as laser power or lens focal length, the results gathered by the camera script will be reliable.

The results from all three tests conducted are relative to the rigs they were performed on, but the trends found in the data will prove to be true when tested on other more precise photophoretic test rigs. The miniature test rigs are not meant to replace other test rigs entirely. Still, they are meant to allow researchers to explore all parameters and possibilities quickly 
and efficiently in edisonian (trial and error) testing to find

trends and discoveries for further research on more precise rigs.

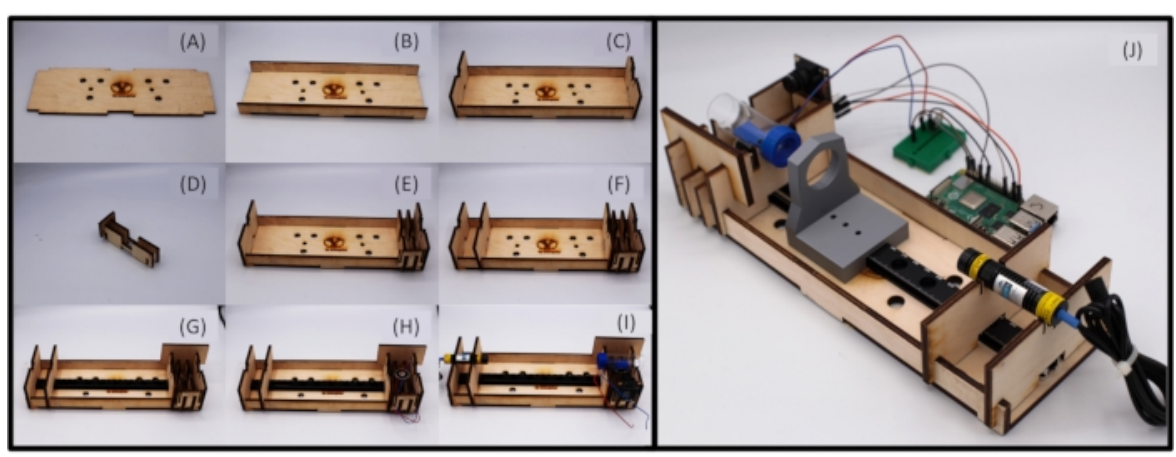

Figure 1: Rig progression with the completed miniature photophoretic test rig. The figure corresponds with step 2 and its sub-steps. (A) Shows step 2.1. (B) Demonstrates step 2.2, the base with the two long sides. (C) Shows step 2.2, the rig's frame, a base with both sides, and the first laser holder and test tube holder. (D) Step 2.3 shows the camera holder combined with both electromagnet holders. (E) Step 2.3.1 offers the combination of Figures 1C,D. (F) Step 2.4, the second test tube holder and the second laser holder have been added. (G) The optional light shield and optical rail have been added. (H) The electromagnet is placed in its holder. (I) The laser and the test tube have been placed in their holders. (J) This shows the entire completed test tig without the power source for the microcontroller board. Please click here to view a larger version of this figure. 


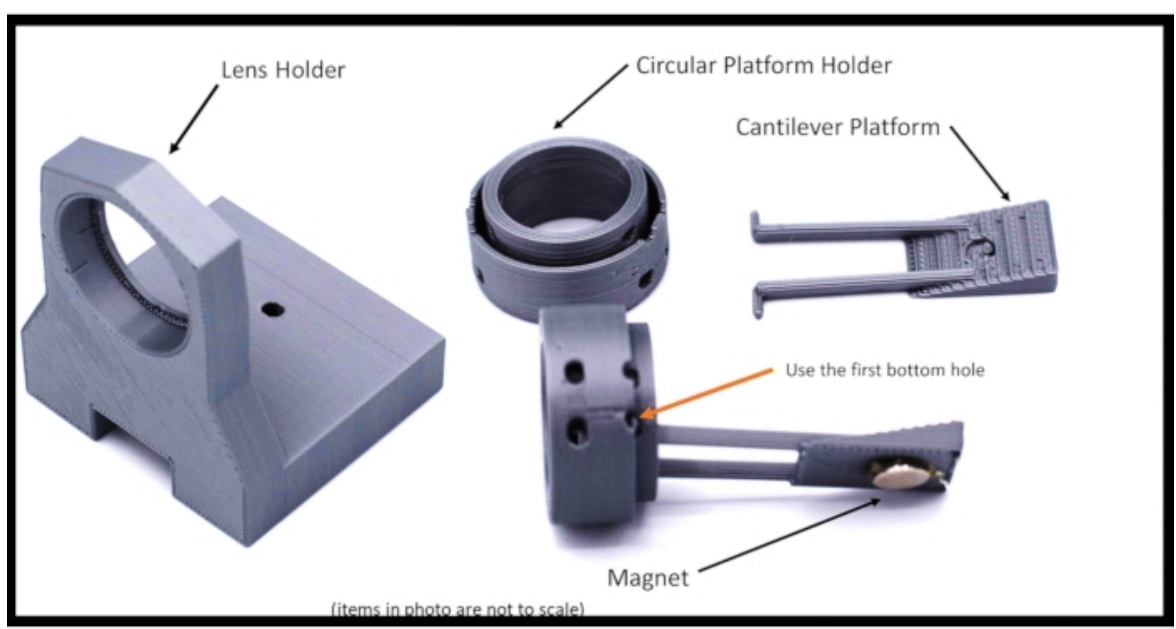

Figure 2: 3D-printed pieces. Included in this figure are the lens holder, circular platform holder, and cantilever platform. The design for the 3D-printed lens holder can be found in Supplemental File 1. This lens holder, when printed, is for a lens of $30 \mathrm{~mm}$ in diameter. Supplementary File 2-3 contain the designs for the platform holder and platform. The platform holder has four sets that the platform can use, but for the rig to work as designed, the platform should use the holes indicated in the figure. Please click here to view a larger version of this figure. 


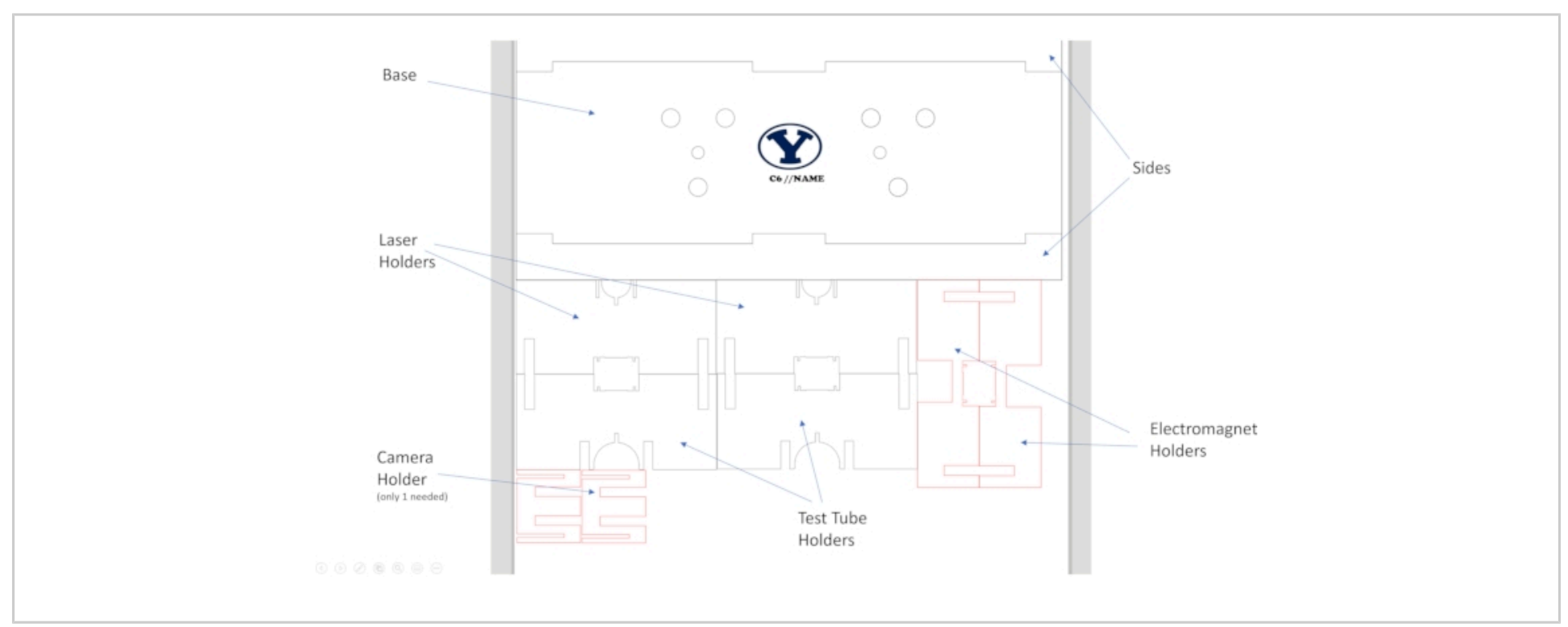

Figure 3: Labeled laser cut pieces. This figure labels the pieces of Supplementary File 4, which contains the file for all the laser-cut pieces except for the optional light shield. After printing, there should be 1 base, 2 sides, 2 laser holders, 2 test tube holders, 2 electromagnet holders, and 2 camera holders (only one is necessary). The optional light shield can be found in Supplementary File 5. Please click here to view a larger version of this figure.

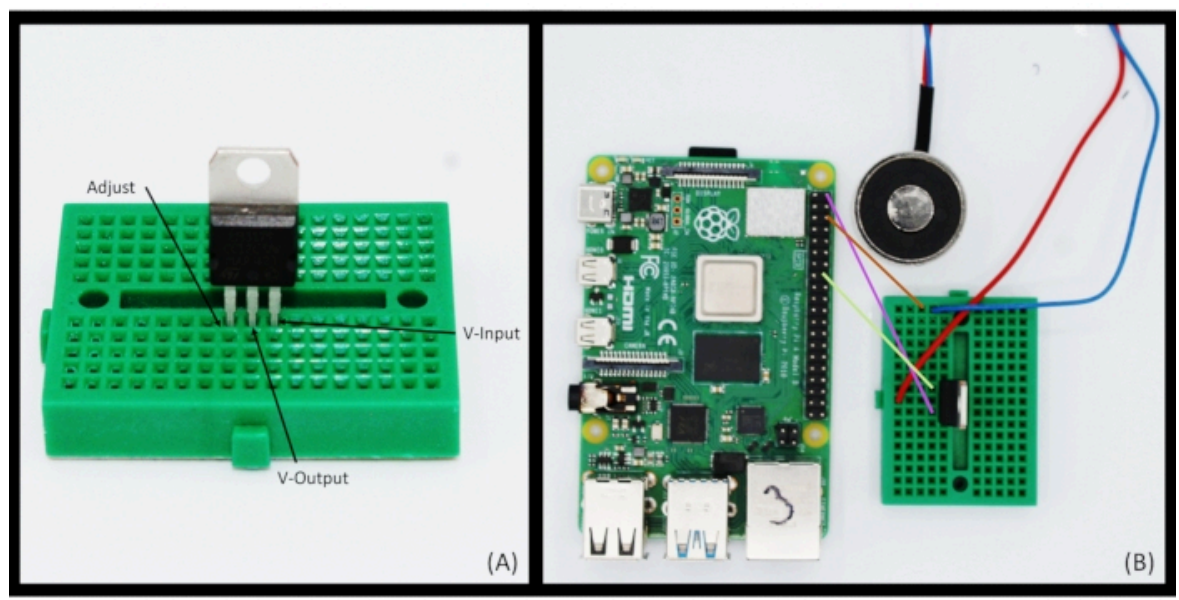

Figure 4: Voltage regulator and electromagnet circuit. (A) For reference, when building the circuit. The voltage regulator has 3 pins, an adjust, input, and output. (B) This figure shows the completed circuit described in step 3. Please click here to view a larger version of this figure. 


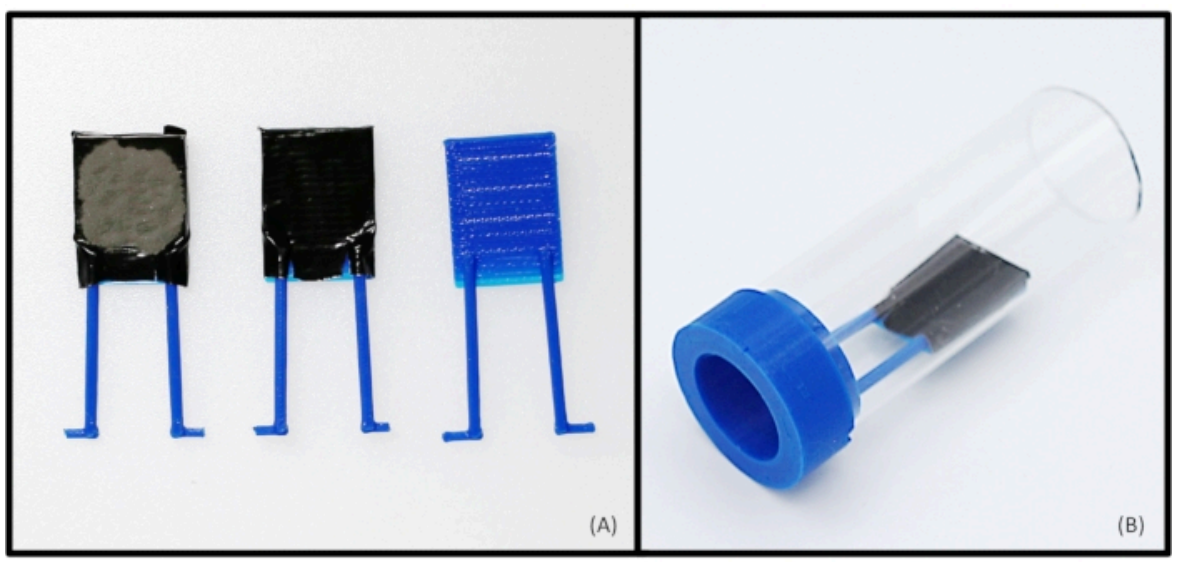

Figure 5: Platform preparations and test-tube trap cite. (A) Before testing can be done, the platform must be prepared. The reservoir of particles where the laser will shine to pick up particles will be placed on the platform immediately before testing. Black aluminum foil should be placed on the platform before the particles. This prevents the laser from melting through the platform. (B) During testing, the actual trapping of particles occurs within the test tube, ensuring consistent automatic motion of the platform for each trap attempt. Please click here to view a larger version of this figure.

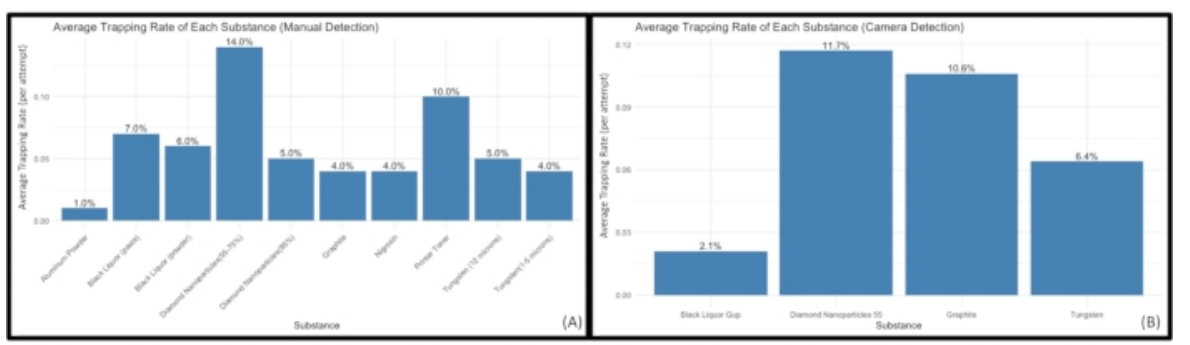

Figure 6: Particle type test (manual) and (camera). (A) A test of 10 different particles was conducted to find the particle with the best trapping rate. (B) A second particle type test was conducted with a camera detection system. Only 4 of the original 10 particles were tested. Please click here to view a larger version of this figure. 


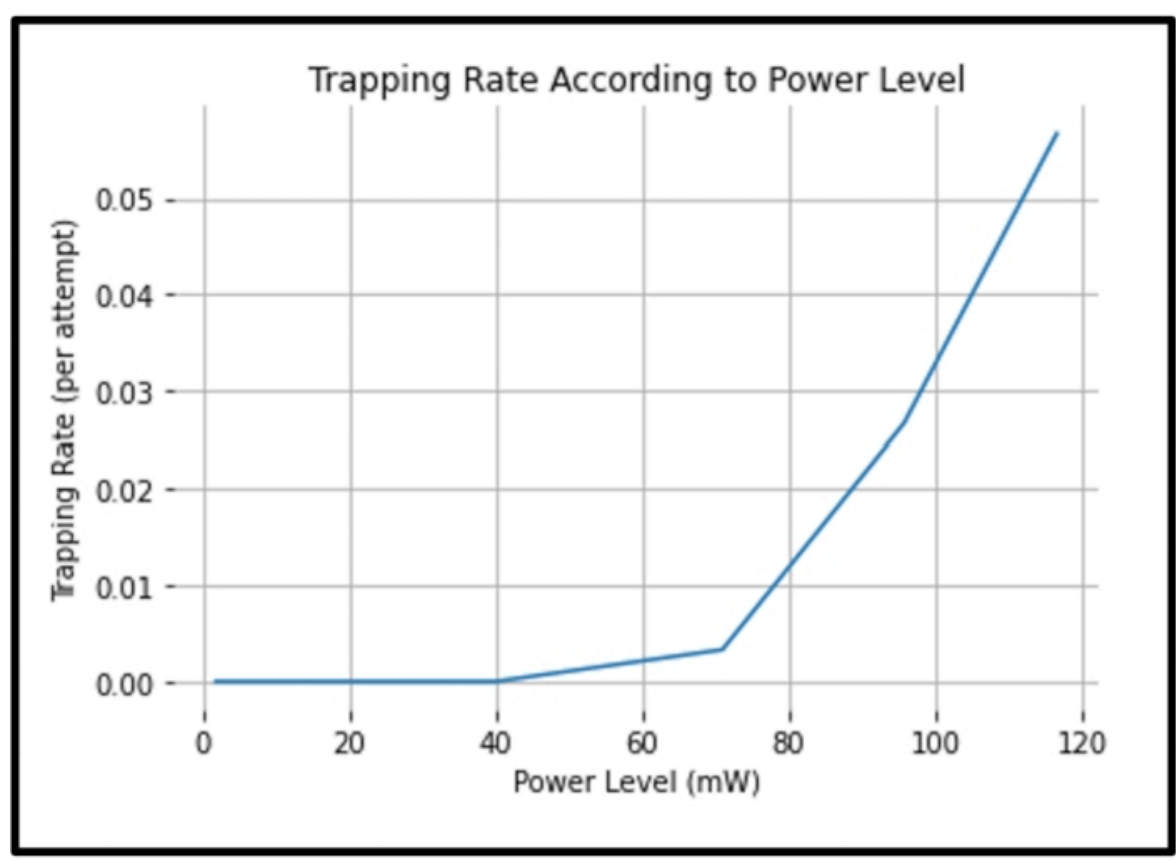

Figure 7: Laser power test results. The rate of trapping for different laser power levels was measured during the laser power test. The higher powers produced higher rates of trapping. Please click here to view a larger version of this figure.

\begin{tabular}{|c|c|c|c|c|c|c|c|c|c|}
\hline $\begin{array}{l}\text { Black } \\
\text { Liquor } \\
\text { (powder) }\end{array}$ & $\begin{array}{l}\text { Black } \\
\text { Liquor } \\
\text { (paste) }\end{array}$ & $\begin{array}{l}\text { Tungsten } \\
\qquad(12 \\
\text { microns) }\end{array}$ & $\begin{array}{l}\text { Tungsten } \\
\qquad(1-5 \\
\text { microns) }\end{array}$ & $\begin{array}{l}\text { Aluminum } \\
\text { Powder }\end{array}$ & $\begin{array}{l}\text { Printer } \\
\text { Toner }\end{array}$ & Graphite & $\begin{array}{c}\text { Diamond } \\
\text { Nanoparticles (95\%) }\end{array}$ & $\begin{array}{c}\text { Diamond } \\
\text { Nanoparticles } \\
(55-75 \%)\end{array}$ & Nigrosin \\
\hline 1 & 2 & 2 & 1 & 0 & 3 & 2 & 1 & 5 & 2 \\
\hline 1 & 3 & 2 & 0 & 1 & 2 & 1 & 2 & 3 & 1 \\
\hline 2 & 1 & 1 & 1 & 0 & 3 & 1 & 1 & 3 & 1 \\
\hline 2 & 1 & 0 & 2 & 0 & 2 & 0 & 1 & 3 & 0 \\
\hline 6 & 7 & 5 & 4 & 1 & 10 & 4 & 5 & 14 & 4 \\
\hline $6.00 \%$ & $7.00 \%$ & $5.00 \%$ & $4.00 \%$ & $1.00 \%$ & $10.00 \%$ & $4.00 \%$ & $5.00 \%$ & $14.00 \%$ & $4.00 \%$ \\
\hline
\end{tabular}

Table 1: Results of the particle type test that would have the best trapping rate. The total sample size of 100 attempts was performed in 4 sets of 25 for each material. 


\begin{tabular}{|c|c|c|c|}
\hline Black Liquor Paste & $\begin{array}{c}\text { Diamond } \\
\text { Nanoparticles } \mathbf{5 5 - 7 5 \%}\end{array}$ & Graphite & Tungsten (12 microns) \\
\hline $2.10 \%$ & $11.70 \%$ & $10.60 \%$ & $6.40 \%$ \\
\hline
\end{tabular}

Table 2: Results of the particle type test conducted with a camera detection system. Data collected from SQLite database. Data was compiled initially in 4 sets of 1000 for a sample size of 4000 per material. Individual records for each set were not compiled from SQLite; only the total percentages were compiled.

Supplementary File 1: File_1-Lens Holder.stl. This contains the 3D printing file for the lens holder (see Figure 2). Please click here to download this File.

Supplementary File 2: File_2-Platform.stl. This contains the $3 \mathrm{D}$ printing file for the cantilever platform (see Figure 2). Please click here to download this File.

Supplementary File 3: File_3-Platform Holder.stl. This contains the 3D printing file for the platform holder (see Figure 2). Please click here to download this File.

\section{Supplementary File 4: File_4-Rig Pieces.odg. This} contains the laser-cutting file for the rig pieces (see Figure 1 and Figure 3). Please click here to download this File.

\section{Supplementary File 5: File_5-Light Shield.odg. This} contains the laser-cutting file for the optional light shield/ blocker. Please click here to download this File.

Supplementary File 6: File_6-Opt1.system.py. This contains the entire code for the use of the instruction from step 4.1. Please click here to download this File.
Supplementary File 7: File_7-Opt2.Read Me.txt. This contains the readme file with a few details for Supplementary Files 8-11. Please click here to download this File.

Supplementary File 8: File_8-Opt2.main.py. This contains the main script for the instructions found in step 4.2. Please click here to download this File.

Supplementary File 9: File_9-Opt2.electromagnet.py. This contains the script for step 4.2 that controls the electromagnet. Please click here to download this File.

Supplementary File 10: File_10-Opt2.test_insert.py. This contains the script for step 4.2 that automatically uploads data into the database. Please click here to download this File.

Supplementary

File 11:

File_11Opt2.camera_controller.py. This contains the script that needs to be uploaded to the camera during step 4.2.2. Please click here to download this File.

\section{Discussion}

The present protocol contains several essential steps that are critical to the automatic running of the trapping rig. First, the electromagnet must be appropriately attached to the 
microcontroller board through the specified circuit. Without the electromagnet, the total utility of the miniature test rig is lost. The electromagnet controls each trapping attempt by raising the particle reservoir on the cantilever platform up into the path of the laser. Each trap attempt is another cycle of raising and lowering the platform.

The camera is only used in step 4.2 as described in the protocol, but it is critical for that option. Step 4.2 requires a camera to detect if a particle has been trapped, allowing data collection from multiple rigs. If the camera is not attached correctly, the rig will not be able to attempt any trapping.

The third and most critical step, step 5.2.1, is aligning and focusing the laser. The lens must be placed so that the focal point occurs over the electromagnet. The cantilevered platform will pass through the focal point over the electromagnet, allowing for particles to trap. Suppose the focal point is not centered above the middle of the electromagnet. In that case, it becomes challenging to ensure that the cantilever platform carrying particles will pass through the focal point to create traps. This can lead to a lack of traps. It is also essential that the platform is elevated over the electromagnet so that the laser path is not constantly contacting the platform. This can cause the camera to report false positives. To more easily adjust the focal point's location, it is suggested to use an optical rail in the setup of the rig; this will allow users to easily slide the lens holder backward or forward to position the focal point properly. The laser and test tube/cantilever part are already aligned if the rig has been appropriately built; the use of the optical rail will keep the lens aligned with the other parts.

Two separate options are detailed in the Protocol, step 4.1 and step 4.2. The first option, step 4.1, is the original simple way to run the miniature tapping rig. This option relies on the human eye to detect particles instead of a camera system. This option is best for collecting smaller sets of data quickly or in situations where a live demonstration is desired. The first option was used during the first two experiments before the second option was created. The second option, step 4.2, uses a camera for automatic detection and trapping, allowing thousands of tests to be run and entered into a database without any human supervision. The accuracy of the camera depends on the exact testing condition; certain more reflective materials, when tested, appeared to have a less accurate trapping rate when compared to similar tests done with human detection. However, several parameters in the camera script can be changed to increase the camera's accuracy. The exact accuracy of the camera is something that can be improved, but it is also not a significant concern because the miniature rigs are meant for initial testing. The second option can also be easily modified to run two test rigs off a single microcontroller board; details for that modification are included in Supplementary File 7.

Current work is developing a more exact and consistent form of automatic trap detection through machine learning. This new machine learning detection system, when finished, will use convolutional neural networks to better detect trapped particles with a much higher rate of accuracy (above 95\%), further bolstering the use and effect such miniature testing rigs can have on the future of photophoretic trap display research.

In its current base form, the miniature trapping rig is limited in a few ways. These miniature rigs are unable to create actual OTDs by scanning the particle after a trap has occurred. The design also limits the possibility of scanners being added for future use in creating OTDs. Another limitation of the design is the need for additional components for a specific test to occur. 
For example, a variable optical attenuator was used to collect the data sets at different optical output power levels during the laser power test. Similarly, if a researcher wished to test laser wavelength in a future test, they would require several other lasers of comparable optical power with different wavelengths in addition to the laser used in this work. The rig would most likely require additional modifications to hold each laser, this process would limit the speed at which such a test could be conducted, but it would still be possible. This design is also determined by the need to $3 \mathrm{D}$ print a new lens holder for each lens. The design and application are also limited to spherical biconvex lenses, which produce spherical aberration to form regions where trapping can occur.

Going forward, future applications include continued testing and optimization of photophoretic trapping parameters. As briefly mentioned above, the miniature trapping rig could easily be modified into a basic inexpensive OTD system by adding scanners for the $y$-axis and $x$-axis control. The electromagnet-controlled particle delivery used in the miniature trapping rig could also be implemented in future advanced OTD systems.

The miniature trapping rig is ultimately unique and distinct in this field of research because it can be inexpensively and quickly fabricated, allowing for rapid mass testing. These rigs are meant to be lean systems designed for the initial testing and optimization of photophoretic trapping parameters. An individual rig can test at a rate of $\sim 250$ attempts per hour. Many other types of photophoretic trapping systems or rigs have been developed to have better automatic systems or accomplish more by scanning the particle to create an image after a successful trap ${ }^{1,8}$. These miniature trapping systems are not meant to replace the use of such systems. They are intended to quickly test parameters and conditions of photophoretic trapping to give researchers a better understanding of what makes for good photophoretic trapping. The miniature trapping rig will democratize photophoretic trap research and allow for a new wave of Edisonian experimentation and progression in this field of research.

\section{Disclosures}

The authors have nothing to disclose.

\section{Acknowledgments}

The authors gratefully acknowledge financial support from the National Science Foundation. NSF Award ID-1846477.

\section{References}

1. Smalley, D. et al. A photophoretic-trap volumetric display. Nature. 553 (7689), 486-490 (2018).

2. Rohatschek, H. Direction, magnitude and causes of photophoretic forces. Journal of Aerosol Science. 16 (1), 29-42 (1985).

3. Desyatnikov, A. S., Shvedov, V. G., Rode, A. V., Krolikowski, W., Kivshar, Y. S. Photophoretic manipulation of absorbing aerosol particles with vortex beams: theory versus experiment. Optics Express. 17 (10), 8201-8211 (2009).

4. Ke, P. C., Gu, M. Characterization of trapping force in the presence of spherical aberration. Journal of Modern Optics. 45 (10) 2159-2168 (1998).

5. Blundell, B. G. On the uncertain future of the volumetric 3D display paradigm. 3D Research. 8, 11 (2017).

6. Smalley, D., Nygaard, E., Rogers, W., Gneiting, S. A., Qaderi, K., Progress on photophoretic trap displays. 
Frontiers in Optics / Laser Science, .OSA Technical Digest. paper FM4C.2. (2018).

7. Rogers, W., Laney, J., Peatross, J., Smalley, D., Improving photophoretic trap volumetric displays. Applied Optics. 58 (34), G363-G369 (2019).

8. Peatross, J., Smalley, D., Rogers, W., Nygaard, E., Laughlin, E., Qaderi, K., Howe, L., Volumetric display by movement of particles trapped in a laser via photophoresis. SPIE Proceedings. 10723, 02 (2018).

9. Smalley, D., Poon, T., Gao, H., Kvavle, J., Qaderi, K. Volumetric Displays: Turning 3-D inside-out. Optics and Photonics News. 29 (6) 26-33 (2018).

10. Shvedov, V. G., Hnatovsky, C., Rode, A. V., Krolikowski, W., Robust trapping and manipulation of airborne particles with a bottle beam. Optics Express. 19 (18), 17350-17356 (2011). 